\title{
El aprendizaje y servicio como realización de la extensión universitaria. El caso de las Prácticas Sociales Educativas en la Universidad de Buenos Aires
}

Oscar Gabriel García

Secretario de Educación Media de la Universidad de Buenos Aires, Argentina.

ogarcia@rec.uba.ar
Gustavo Galli

Secretario de Extensión Universitaria de la Universidad de Buenos Aires. gjgalli@rec.uba.ar
Integración de la docencia y la extensión /

Desafíos de gestión

RECEPCIÓN: 24/06/16

ACEPTACIÓN FINAL: 07/09/16

\section{Resumen}

A partir del año 2017 las Prácticas Sociales Educativas (PSE) tendrán un carácter obligatorio en la Universidad de Buenos Aires. Esta decisión apunta a promover un importante cambio en la formación universitaria. Analizamos su origen, definición y los problemas relacionados con su incorporación curricular. Planteamos la extensión como una forma de relación entre la universidad y la sociedad, donde la estrategia pedagógica que surge son el aprendizaje y servicio solidario como elementos de formación integral de los futuros profesionales. Será entonces que, mientras el aprendizaje y servicio le dan contenido teórico a la extensión universitaria, las PSE realizan esta tradicional misión del sistema de educación superior en la Argentina. Presentaremos entonces el modelo de implementación de estas prácticas educativas en la Universidad de Buenos Aires.

Palabras clave

- Extensión universitaria

- Educación situada

- Aprendizaje y servicio

- Prácticas Sociales Educativas

- Impacto curricular

\section{Resumo}

A partir do ano 2017, as Práticas Sociais Educacionais (PSE) devem ter a condição de obrigatórias na Universidad de Buenos Aires. Esta decisão tenta promover uma mudança significativa na formação universitária. Analisaremos a origem, a definição e os problemas relacionados com a sua incorporação curricular. Consideramos a extensão curricular como uma forma de relação entre a universidade e a sociedade, em que a estratégia pedagógica que surge é a aprendizagem e o serviço solidário como elementos de formação integral dos futuros profissionais. Nesse sentido, enquanto a aprendizagem e o serviço realizado dão conteúdo teórico à extensão universitária, as PSE realizam esta tradicional missão do sistema de educação superior na Argentina. Apresentaremos o modelo de implementação destas práticas educacionais na Universidade de Buenos Aires.

Palavras-chave

- Extensão universitária

- Educação em contexto real

- Aprendizagem e serviço

- Práticas Sociais Educacionais

- Impacto curricular

\section{Para citación de este artículo}

García, O. y Galli, G. (2016). El aprendizaje y servicio como realización de la extensión universitaria. El caso de las Prácticas Sociales Educativas en la Universidad de Buenos Aires. En Revista +E versión digital, (6), pp. 104-111. Santa Fe, Argentina: Ediciones UNL 


\section{Introducción}

Durante los últimos años, nuestro sistema de educación superior ha instrumentado acciones tendientes a jerarquizar las actividades por las cuales la universidad se vincula con la comunidad. La implementación de propuestas que relacionan a las universidades con el medio en que se desarrollan, como la creación del Programa de Fortalecimiento de la Universidad Argentina por parte del Ministerio de Educación en 2010, sumada a la creación, en julio de 2008, de la Red Nacional de Extensión Universitaria (REXUNI), en su condición de comisión asesora del Consejo Interuniversitario Nacional (CIN), serían solo algunas de las propuestas que confirman esta tendencia.

Hoy la Universidad de Buenos Aires (UBA) cuenta con un programa de subsidios para proyectos de extensión universitaria consolidado y en permanente crecimiento, ${ }^{1}$ con un aumento considerable en las becas de ayuda económica y el desarrollo del primer programa integral e interdisciplinario de acción comunitaria en barrios vulnerables (Resol. CS n 4308/2008). De esta manera orienta gran parte de sus acciones hacia la comunidad de la cual se nutre. En este sentido, la implementación de las Prácticas Sociales Educativas $(\mathrm{PSE})^{2}$ constituye la última etapa de una clara política pública que tendrá como fin el de generar mecanismos para poner al servicio de la sociedad los saberes que allí se producen y enseñan.

"La universidad tiene que convertirse en uno de los factores principales del cambio profundo que exige la situación actual.

Para lograr este objetivo, es imprescindible conocer y responder a las necesidades, requerimientos y aspiraciones de la comunidad" (Risieri Frondizi, 1971:247).

Ahora bien, ¿cómo se debe proceder? ¿De qué hablamos cuando nos referimos a vincular la institución universitaria con la comunidad? ¿Son la "educación situada o experiencial", el "aprendizaje y servicio" y/o las PSE formas en la que la extensión universitaria se realiza? ¿Es la extensión universitaria lo suficientemente explícita a la hora de definir la función social de las universidades? Seguramente no. La extensión, como una de los tres pilares en que se funda nuestro sistema de educación superior, no ha adquirido en el tiempo los atributos esenciales que la caractericen exhaustivamente respecto de la docencia y la investigación científica. Esto ha dado origen a un gran problema de gestión debido a que la ha transformado en un área de actividades compleja y abarcativa que intenta explicar un sinnúmero de cuestiones hacia el interior del sistema de educación superior sin anclar en ninguna de ellas. Es que esta noble misión se ha desarrollado sin lineamientos estratégicos sostenidos, lo que le imposibilitó construir un cuerpo teórico donde apoyarse para continuar su tarea, acumular experiencias, perfeccionar prácticas y generar el debate sobre los problemas socioculturales de la actualidad.

Sumergirse en el debate de las propuestas de las prácticas sociales es asumir el desafío de abordar un tema sumamente complejo, no por el tipo de prácticas al que se estaría refiriendo sino, y fundamentalmente, al mismo concepto de práctica universitaria tan discutido durante los últimos años. Es que la separación entre enseñanza teórica y práctica lleva más de siglo y medio sin llegar a conclusiones categóricas, sobre todo en Argentina, cuyo sistema de educación superior se caracteriza por ser profundamente academicista. Sin embargo, la pedagogía está haciendo un giro que permite una mirada renovada sobre la "práctica" y posibilita a los alumnos realizar su aprendizaje de modo más efectivo.

Tradicionalmente, el proceso de enseñar y de aprender corría por la misma avenida: era común ver a los especialistas en didáctica hablar de proceso de enseñanza-aprendizaje como si se tratara de un proceso continuo donde la transmisión de conocimiento se jugará solo en la primera parte del término. En pocas palabras, si se controlaban todas las variables de la enseñanza automáticamente los alumnos aprendían. $Y$ si no ellos fracasaban.

Por eso, la enseñanza es concebida como un proceso complejo que se desarrolla en contextos institucionales de incertidumbre y donde el docente opera a partir de supuestos personales y condiciones institucionales que muchas veces no controla y que determinan fuertemente sus resultados (Davini, 1993). Enseñar, educar, supone la intencionalidad de transformar, de producir cambios en aquellos que son el centro de la acción educativa y, sobre todo, de seleccionar las estrategias pedagógicas para tal fin, el que sumado a una adecuada infraestructura hará de la enseñanza una actividad dirigida y efectiva a la hora de transmitir. Pero ello, ¿es suficiente? Seguramente no. El término complementario de "aprendizaje" alude tanto al proceso mediante el cual se adquiere un conocimiento (tarea) como a su incorporación efectiva (rendimiento). Entonces la enseñanza incide sobre el aprendizaje como tarea, y son las tareas desarrolladas por el alumno, las responsables del aprendizaje como rendimiento (Basabey Cols, 2004). Así, tanto la enseñanza como el aprendizaje no pueden ser vistos como etapas de un mismo proceso lineal debido a que son dos procesos separados y diferentes, ya que una cosa es enseñar y otra muy distinta es cómo
1) Programa de subsidios UBANEX que con la Resolución (CS) $n^{\circ} 3411 /$ 2015 aprobó el 8vo. llamado.
2) Programa de Prácticas Sociales Educativas - Resoluciones (CS) $n^{\circ} 520 / 2010$ y $3653 / 2011$. 


\section{4}

cuando se piensa en las prácticas de

aprendizaje y servicio debe pensarse

en una innovación pedagógica que

determina un proceso formativo integral el alumno se apropia de lo que se le enseña. Por supuesto que no existe aprendizaje sin enseñanza ni enseñanza sin aprendizaje, ciertamente son dos procesos diferentes pero conjugados. En resumen, solo en la experiencia subjetiva del alumno se realizará el aprendizaje puesto que permitirá en él elaborar las estructuras cognitivas por las cuales se fijan los conocimientos.

En este sentido, cuando se piensa en las prácticas de aprendizaje y servicio debe pensarse en una innovación pedagógica que determina un proceso formativo integral, de modo bidireccional entre teoría y experiencia práctica. Implica además aprendizajes activos y significativos centrados en el sujeto que aprende y una concepción del conocimiento como bien social. Y, sobre todo, donde las prácticas didácticas estén dirigidas a capacitar a los docentes en la difícil tarea de integrar la enseñanza y la investigación y al mismo tiempo desarrollar en los jóvenes habilidades sociales. (Campus Compact, 1999).

Para los defensores de esta corriente pedagógica, se establece que las instituciones educativas no solo tienen la responsabilidad de desarrollar en los estudiantes los conocimientos, competencias y aspiraciones de realización personal, sino además la de buscar que éstos se comprometan con el contexto de la comunidad en la cual se desarrollan.

El presente trabajo intentará echar luz sobre los problemas mencionados, posibilitar la reflexión sobre las diferentes corrientes y teorías en que se realiza la extensión universitaria y poner énfasis en el aprendizaje y servicio, la implementación de las PSE y el impacto en la formación y rendimientos de los alumnos que participan de las mismas.

\section{La conceptualización del aprendizaje y servicio}

El largo recorrido de la extensión universitaria como acción formativa tiene sus orígenes en las universidades británicas de fines del siglo XIX. Allí donde las consecuencias de la Segunda Revolución Industrial hacían necesario capacitar a los empobrecidos obreros que debían estar a cargo del proceso de producción en masa, las universidades rompieron el gueto que protegía las mentes prístinas de sus alumnos e instrumentaron los primeros cursos extracurriculares para trabajadores de las industrias textiles. Poco a poco esta noble misión se fue extendiendo hacia todo el ámbito laboral haciéndose más abarcativa y cubrió incluso necesidades que iban más allá de la capacitación. Esta forma de abordaje general de los problemas sociales fue la que influyó a las universidades hispanoamericanas y posibilitó la generación de innumerables proyectos elaborados desde diferentes disciplinas académicas. Las labores extensionistas así concebidas se definirían como las acciones directas e inmediatas en el campo social, apoyadas en una matriz de investigación y docencia. La investigación permite que abordemos el campo con conocimiento de las verdaderas causas del problema social, como también que planifiquemos las acciones a futuro (metodología y objetivos), mientras que la docencia forma a aquellos que deberán intervenir en el territorio, puesto que no de cualquier modo se llevará adelante dicha intervención (actividades y tareas). En este sentido, podemos establecer que toda práctica extensionista conlleva la necesidad de poner el foco tanto en la comunidad como en la formación personal de aquellos que la ejercen. Es por ello que en las últimas décadas ha aumentado la oferta de medidas formativas que tomen en consideración el desarrollo de una cultura democrática y participativa, que despierten el interés de formar parte activa de la vida pública y que promuevan la convivencia en sociedades multiculturales desde la comprensión y el diálogo, luchando contra la exclusión en pro de la cohesión social (Folgueiras Bertomeu, 2013).

Es importante destacar que en el aspecto pedagógico son varias las propuestas que se han desarrollado, pero tal vez una de las que con más fuerza ha avanzado es la del aprendizaje y servicio (Ap-S). Prácticamente todas las universidades norteamericanas disponen de programas de Ap-S. También puede verificarse la importancia de diversas fundaciones privadas que dan soporte a iniciativas y programas de Ap-S, como Kellogs, Carnegie, ReadersDigest, etcétera. 
Al mismo tiempo, la expansión de los proyectos de aprendizaje y servicio en Latinoamérica, particularmente en países como Argentina, Uruguay o Chile, sale de la tradicional práctica profesional y se asocia a una vocación de ayuda social realizando servicios comunitarios de transferencia directa; de aquí que se prefiera hablar más de Aprendizaje y Servicio Solidario que de Aprendizaje y Servicio. Las primeras prácticas se sitúan en los años ochenta, pero será en el año 2000 que estas propuestas se consolidan con la creación de Centro Latinoamericano de Aprendizaje y Servicio Solidario (CLAYSS) en Buenos Aires. Son muchas y variadas las definiciones que se han expresado en nombre del aprendizaje y servicio. Cada una de ellas ceñida por la naturaleza de la institución educativa y el contexto en que se desarrollan las actividades. En tanto, se pueden observar dos grandes tendencias: una del origen anglosajón, que entiende el Ap-S (Learning-Service) como una de las tantas formas de aprendizaje a través de la experiencia, del "aprender haciendo". En esta línea, el énfasis estará puesto en el desarrollo de habilidades técnicas más allá de las consecuencias o impacto de dichas prácticas. Aquí el vocablo service evoca más el "hacer juntos" que el "dar para" (Tapia, 2003). Por el contrario, en el ámbito iberoamericano el concepto de "servicio" se presenta más extensivo, entendiéndose como una respuesta solidaria a los tantos desafíos que plantea la condición de vulnerabilidad social. Será por ello que la difusión en Latinoamérica se ha dado bajo la denominación de "aprendizaje y servicio solidario". Otro de los conceptos utilizados con el fin de describir estas prácticas suele ser la "educación situada", que considera el aprendizaje escolar como un proceso en el que los estudiantes se integran de manera gradual a una comunidad de prácticas sociales. Es la enseñanza entendida como producto de la experiencia, así la educación experiencial suele ser denominada comúnmente como enseñanza situada. En este caso, se refiere a una clase particular de aprendizajes, a una estrategia de enseñanza con enfoque holístico que está destinada a relacionar el aprendizaje académico con la vida real (Camillioni, 2009). Por lo tanto, para la enseñanza situada aprender y realizar actividades para y con la comunidad son acciones inseparables, por lo que los alumnos han de aprender haciendo dentro del contexto pertinente (Díaz Barriga, 2003). Algunas de las estrategias de enseñanza centradas en el aprendizaje experiencial y/o situado, son las siguientes:

- Enfocan la construcción del conocimiento en contextos reales, a través de proyectos participativos y análisis de casos. También en la construcción de conocimientos y habilidades para diseñar planes y proyectos de acción social en los que intervengan de manera sustantiva los estudiantes.

- Hacen hincapié en el desarrollo de las capacidades reflexivas, críticas, y en el pensamiento de alto nivel.

- Se centran en la participación en prácticas sociales auténticas de la comunidad y fomentan el trabajo en equipo de tipo colaborativo.
Respecto de estas estrategias cabe señalar dos cosas:

1. Algunas de estas estrategias han sido desarrolladas hace décadas y tienen sus antecedentes en la enseñanza experiencial, el método de proyectos y el análisis de casos. Sin embargo, ahora están siendo reconceptualizadas desde la perspectiva situada y sociocultural.

2. Varias de estas estrategias pueden combinarse en la práctica e incluso encontrarse integradas en propuestas de aprendizaje y servicio.

Cabe mencionar que en la enseñanza situada la unidad básica de análisis no es el individuo ni los procesos de aprendizaje propiamente dichos sino que la acción recíproca, es decir, la actividad relacional de las personas que actúan en contextos determinados. De esta manera, una situación educativa, a efectos de su análisis e intervención instruccional, requiere ser concebida como un sistema de actividad cuyos componentes incluyen:

- El sujeto que aprende.

- Los instrumentos utilizados en la actividad.

- El objeto a apropiarse u objetivo que regula la actividad (saberes y contenidos).

- Una comunidad de referencia en que la actividad y el sujeto se insertan.

- Normas o reglas de comportamiento que regulan las relaciones sociales de esa comunidad.

- Reglas que establecen la división de tareas en la misma actividad.

De lo anterior se desprende que la enseñanza situada se constituye como síntesis entre las líneas de trabajo anglosajón y la latinoamericana, por lo que originan una novedosa pedagogía que establece un modo específico de vincular la institución universitaria y la comunidad. Un modo por el cual se deben construir espacios de reflexión y aprendizaje curriculares que ayuden a comprender la realidad y a desarrollar capacidades creativas para enfrentar nuevas situaciones que den respuestas a entornos sociales problemáticos. Estos espacios actuarán como interfaces bidireccionales donde los profesores y alumnos aprenden y la comunidad se beneficia con la trasmisión de conocimiento.

Más recientemente, estas propuestas se han ido difundiendo en Europa. Los primeros intentos de sistematización se sitúan ya iniciado el siglo XXI con la creación de distintas organizaciones, programas y encuentros. Algunos ejemplos que cita Folguiras Bertomeu (2013) en la actualidad son los siguientes:

- 2002, Higher Education Active Community Fund (Gran Bretaña).

- 2005, Service Learning: Dialogue between Universities and Communities (Proyecto Leonardo: CIVICUS) (Unión Europea).

- 2005, Forum Cívico-Educativo (Madrid, España).

- 2005, Centro Promotor de Aprendizaje-Servicio (Barcelona, España). 
En el caso de la UBA, es importante destacar el Programa Integral de Acción Comunitaria en Barrios Vulnerables creado por el Consejo Superior de la UBA en 2008, que fue premiado en distintas ocasiones por el desarrollo de las buenas prácticas en Ap-S. Entre las distinciones obtenidas se cuentan:

- Declaración de Interés Educativo por la Legislatura de la ciudad de Buenos Aires. Proyecto aprobado en junio de 2010.

- Mención especial del Premio Presidencia de la Nación a las "Prácticas Educativas Solidarias en Educación Superior 2010" para el Programa Integral de Acción Comunitaria en Barrios Vulnerables de la Secretaría de Extensión Universitaria y Bienestar Estudiantil de la UBA, agosto de 2010.

- Primer lugar en el premio Mac Jannet Prize For Global Citizenship (premio a la ciudanía global) otorgado por la Red Talloires:

asociación internacional que fomenta que las universidades incorporen la participación ciudadana y el servicio a la comunidad en su misión de investigación y enseñanza 2011.

\section{Las PSE: el caso de la UBA}

En la formación superior es de radical importancia reflexionar sobre las competencias profesionales que el contexto requiere. Hoy la realidad laboral demanda una formación integral que, en resumidas cuentas, no puede significar solo la adquisición de contenidos teóricos ni de habilidades técnicas sin que ellas se hayan puesto a prueba en escenarios reales en donde luego se irán a desarrollar. Por lo expuesto, plantear una nueva enseñanza significará brindar herramientas para que los alumnos obtengan diversas visiones del mundo, participen en nuevas y variadas formas de las prácticas educativas y adquieran una posición reflexiva frente a la realidad. Eso debe constituirse en el objetivo esencial de una instrucción

\section{Cuadro de clasificación} de estrategias de educación experiencial

\begin{tabular}{ll}
\hline Pasantía & $\begin{array}{l}\text { Práctica educativa donde el foco se pone solo en la } \\
\text { formación técnica individual. }\end{array}$
\end{tabular}

Solidarias

Prácticas educativas que ponen el acento solo en el beneficio a la comunidad.

Voluntariados

Prácticas educativas en que el foco se pone tanto en el beneficio a la comunidad como en el aprendizaje de conocimientos no curriculares.

Aprendizaje-servicio

Práctica social en que el foco se pone tanto en el beneficio a la comunidad como en el aprendizaje de conocimientos curriculares.

Fuente: elaboración personal sobre la base de las apreciaciones realizadas por la profesora Alicia Camilloni (2013). superior integral, donde a la adquisición de conocimientos específicos se sume la capacidad de poder comprender, compartir y producir significados, adoptando una actitud activa frente a la realidad social por medio de una mirada reflexiva, crítica y creativa. El reto aquí es desarrollar tareas en la comunidad que puedan transformarse en experiencias de aprendizaje dignas del curso académico con el que se integran. La educación experiencial o situada es la que se produce fuera del aula y en vínculo con la comunidad. Implica necesariamente una interacción. Integra aprendizaje y desarrollo personal (Kolb, 1984).

Un modelo explicativo de la educación experiencial o situada nos permite ver con más claridad la configuración de diversas estrategias pedagógicas. Este modelo presenta cuatro cuadrantes que resultan de aplicar dos criterios: por un lado, la relación del estudiante con la comunidad (servicio social) y, por otro, el vínculo del estudiante con los aprendizajes establecidos en el currículo (instrucción formal).

Cuando las prácticas educativas como estrategia pedagógica se sustentan en reconocer y satisfacer intereses de la comunidad, sin estar relacionado ello con el currículo, estamos frente a actividades netamente solidarias. Si reconocen y satisfacen intereses curriculares (de adquisición de competencias técnicas) sin estar relacionadas con la acción directa en la comunidad, nos referimos a las clásicas pasantías como práctica profesional. Es importante destacar en este caso que la comunidad es un escenario pasivo en el que poco importan las modificaciones que operen en él. Por otra parte, si la actividad se enmarca en un servicio social y el estudiante aprende pero lo hace en cuestiones no relacionadas con el currículo, estamos en presencia de los llamados voluntariados. Y por último, si la estrategia pedagógica implica la conjugación total de ambas dimensiones, es decir, un alto grado servicio social y alta relación con los mandatos curriculares, se trata de lo que se conoce como aprendizaje y servicio. Por supuesto que este tipo de estrategia requiere ampliar los contenidos curriculares a la socialización de valores y competencias, tales como desarrollar en el estudiante la capacidad de pensamiento crítico y plantear posibles soluciones a cuestiones sociales. El estudiante será entonces capaz de identificar y definir problemas, de usar tecnologías con propósitos y necesidades propias y no ser usado por ellas, capaz de actuar con autonomía en situaciones inesperadas, de enfrentar encrucijadas y tomar decisiones con sentido ético y de trabajar por un nuevo y mejor mundo guiado por las relaciones entre el conocimiento objetivo, el propósito moral y la práctica social.

En este sentido, debemos destacar que las prácticas sociales universitarias y las prácticas profesionales difieren en varias cuestiones. Si bien es cierto que ambas son formas de aprendizaje basado en la experiencia, son marcadamente diferentes. En primer lugar, como ya vimos, las PSE privilegian tanto la adquisición de 


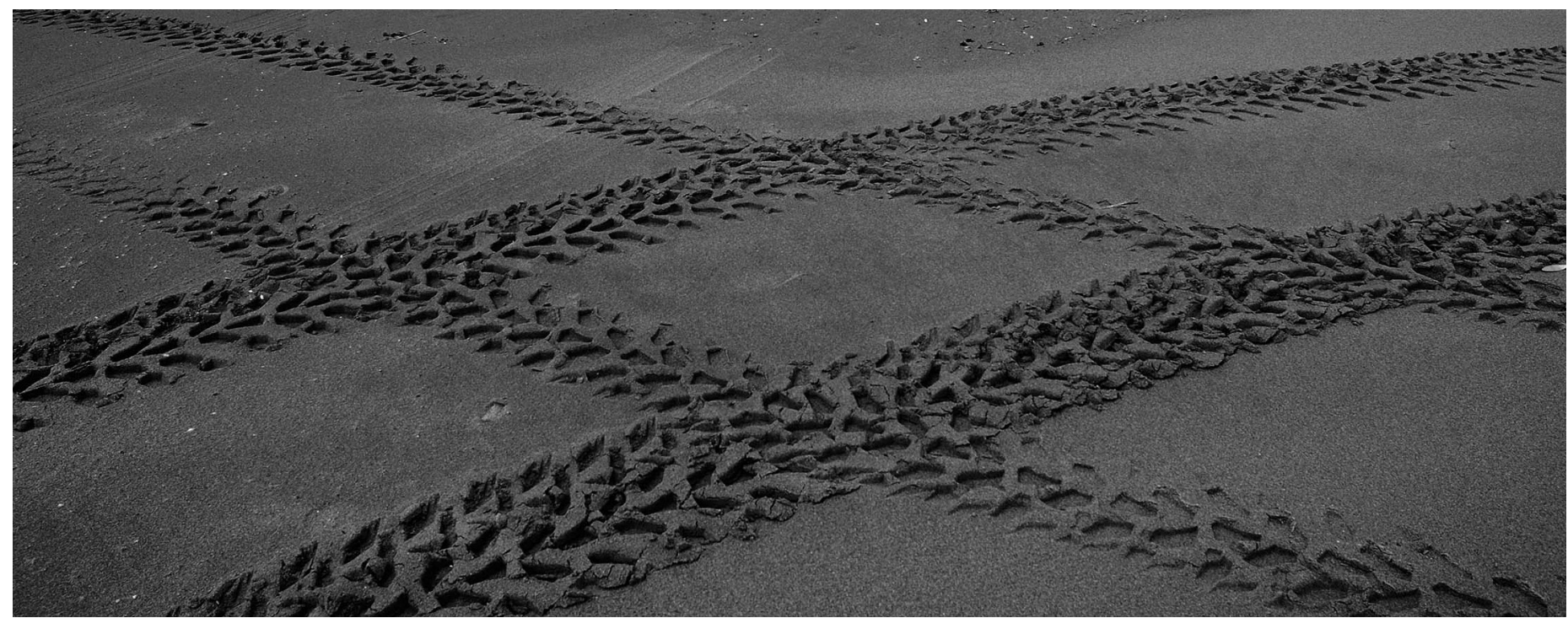

(C) Oscar Dechiara

conocimientos como el beneficio concreto de este conocimiento en el campo social, mientras que la pasantía o práctica profesional se centra solo en el servicio brindado como entrenamiento para el alumno (Honnet-Porter \& Poulsen, 1989). En segundo lugar, las prácticas profesionales están impulsadas por las necesidades del plan de estudios; mientras que las PSE, por necesidades curriculares y de la comunidad. En tercer lugar, las prácticas profesionales preparan técnicamente a los estudiantes para su posterior actividad laboral, mientras que las PSE los preparan para un ejercicio profesional ético y responsable frente a la realidad social de su tiempo.

A partir de lo anterior, entendemos como PSE a las actividades de aprendizaje y servicio por las que se logran claros beneficios tanto para la formación individual como para la comunidad en la cual se interviene, promoviendo la integración social con el fin de transformar la realidad en pos de la inclusión, profundizar el desarrollo local y abrir los recursos en poder de la comunidad. Todo ello mediante el trabajo interdisciplinario, intersectorial y participativo, profundizando el sentido integral de las acciones de políticas sociales y contribuyendo así al mejoramiento de la formación individual y de la calidad de vida de las personas. ¿Qué se aprende? Formación académica, habilidades técnicas, responsabilidad ciudadana, responsabilidad en el trabajo, formación ética. El aprendizaje-servicio requiere asociación con la comunidad, objetivos en respuesta a necesidades de la comunidad, objetivos de aprendizajes curriculares explícitos, reflexión, evaluación o tutoría. Se pueden señalar como características del aprendizaje-servicio (Eyler \& Giles, 1999) que: las experiencias son auténticas, implican cooperación más que competencia con otras personas, requieren compromiso personal con la comunidad y actitud ciudadana, se hace necesario tratar con problemas complejos, en situaciones reales, y demandan aprendizaje de nuevos conocimientos. No es suficiente contar con conocimiento abstracto y general, sino que hay que aprender a aplicarlo en situaciones reales. Promueve aprendizajes profundos porque hay que reorganizar el conocimiento adquirido e integrar nuevo. Facilita la evaluación porque los resultados son inmediatos y visibles para los diferentes actores. Compromete a la totalidad de la persona, intelectual, afectiva y físicamente.

Ahora bien, ¿se puede construir una cultura ciudadana responsable? El tema adquiere real importancia debido a que hoy la ciudadanía se ha transformado en un concepto declaradamente difuso. Es claro, si hay algo que ha caracterizado a la modernidad (sobre todo durante el siglo $\mathrm{XX}$ ) es que el concepto de ciudadano era un modelo visiblemente establecido en todos los países por igual. Un ciudadano era aquel individuo que manifestaba ejercer los derechos propios de la condición de ciudadanía en cada nación, y debe ser por eso que se lo suele describir hoy como "ciudadanía estatutaria". Esta tradicional concepción del ciudadano, aferrado al entramado jurídico de un Estado nacional asentado por otra parte, en un ámbito territorial determinado, es lo que en los últimos 40 años ha entrado en crisis, fundamentalmente por los sucesivos cambios en el escenario internacional: los flujos migratorios masivos, la creciente multiculturalidad, la sociedad de la información, el impacto tecnológico en las comunicaciones, las políticas de género, el avance en la legislación de los jóvenes, etc. Se fue erosionando poco a poco esta idea tradicional de ciudadanía. Hoy hablamos de ciudadanía cosmopolita, que incluso pone en crisis la misma definición de Estado nacional y hace de ella algo indeterminado y flexible. 
Dar respuesta educativa a estas nuevas dimensiones de ciudadanas y ciudadanos es entonces todo un desafío y se ha convertido en la base de los debates intelectuales de principio de siglo XIX. Esta novedosa concepción de "ciudadanía" se entiende en el ejercicio pleno de los derechos y no en la descripción jurídica objetiva; por lo tanto, el ciudadano es aquel que se "empodera" a partir de la participación activa que garantiza la realización de los derechos. El nuevo modelo de ciudadanía pasa a ser un proceso de construcción de todos los individuos participantes, por lo cual no se define de modo acabado y debe ser construido todos los días, haciendo de la educación un instrumento extraordinario para tal fin (Folgueiras Bertomeu, González y Julián, 2010).

\subsection{Los objetivos de las PSE}

Como objetivo central se propone lograr integralidad en el proceso de aprendizaje, con impacto curricular, a partir de prácticas interdisciplinarias y obligatorias, y asimismo desarrollar en los alumnos la cooperación y la inteligencia colectiva.

Entre los objetivos específicos, las PSE se plantean:

- Implicar a los alumnos en una experiencia "auténtica", fuerte y común, para volver a ella de una manera reflexiva y analítica, fijando nuevos saberes.

- Promover la integración social con el fin de transformar la realidad en pos de la inclusión, profundizar el desarrollo local y ampliar los recursos en poder de la comunidad a partir del trabajo interdisciplinario, intersectorial y participativo.

- Profundizar el sentido integral de las acciones de políticas sociales y mejorar la formación individual y de la calidad de vida de las personas.

- Promover el desarrollo de valores de ciudadanía fundados en el compromiso social.

- Potenciar la vinculación de las prácticas académicas universitarias con la comunidad en la que se inserta la institución articulando las necesidades curriculares y de la comunidad.

- Integrar las funciones de enseñanza, investigación y extensión.

- Posibilitar miradas reflexivas y críticas sobre la sociedad desde los enfoques propios de las disciplinas.

- Desarrollar la cooperación y los vínculos entre docentes y estudiantes, y entre ellos y la sociedad.

- Incentivar el desarrollo del pensamiento crítico y su utilización para la solución de problemas concretos.

- Desarrollar la autonomía y la capacidad de hacer elecciones y negociarlas.

- Formar para la concepción y la conducción de proyectos. Liderazgos institucionales.

\subsection{Organización y gestión operativa}

Las PSE, que comenzaron a implementarse en los currículos de todas las carreras en condición de optativas durante los ciclos lectivos 2012/2016, serán obligatorias a partir del año 2017. Todas las Facultades elevarán al Consejo Superior de la UBA una lista con los proyectos que se ofrecerán a los estudiantes mediante una base de datos establecida a tal fin, incluyendo entre otros datos el cupo de alumnos permitido y el cronograma de tareas. Los estudiantes podrán comenzar las PSE una vez finalizado el Ciclo Básico Común y el primer cuatrimestre de la carrera y podrán optar por los proyectos ofrecidos por su Facultad de origen o de otra unidad académica. Estas prácticas tendrán una duración cuatrimestral de 42 horas como mínimo y estarán supervisadas por un profesor y un equipo de auxiliares docentes que guiará las actividades. Los estudiantes deberán llevar adelante y por escrito un relevamiento de todas las actividades semanales a modo de bitácora, las cuales deberán ser firmadas por el docente a cargo del proyecto. Al finalizar las prácticas, los profesores a cargo emitirán un certificado para cada alumno que haya aprobado dichas actividades. En el caso de alumnos que realicen las PSE en proyectos que no pertenezcan a su unidad académica, el certificado deberá ser firmado, además de por el director general de la práctica, por la Secretaría Académica de la Facultad de donde dependa el proyecto.

Vale recordar que una PSE constituye un trayecto formativo que incluye tres componentes: uno formativo, que supone el desarrollo de habilidades mediadas a través de procesos de enseñanza, uno social en la medida en que es una práctica que se desarrolla en espacios extraáulicos y se dirige a la sociedad, y un componente de intervención por cuanto implica el desarrollo de estrategias con el objetivo de brindar un servicio a la comunidad en la que se inserta la institución.

\section{A modo de conclusión}

Hasta aquí se ha intentado realizar una aproximación a la necesidad de revalorizar las prácticas educativas en educación superior como forma de lograr aprendizajes más efectivos y a su definición y los problemas que surgen relacionados con la incorporación curricular de las mismas. Además, se abordó la manera en que dichas actividades curriculares se presentan en estrategias pedagógicas que, en la interacción con la comunidad, permiten desarrollar tareas con las cuales el alumno aprende cuestiones que se encuentran más allá de las competencias técnicas, tales como habilidades sociales, formación ética y responsabilidad ciudadana.

No debe pensarse entonces el compromiso social de la universidad como la mera transferencia de conocimientos y tecnologías que produce, sino que se hace imprescindible comprender las necesidades comunitarias como punto de partida para la creación de nuevos saberes, con la certeza de que el abordaje de las situaciones críticas que se generan en los espacios populares cambian actitudes y producen a su vez conocimientos que actúan como factores determinantes de la madurez y el compromiso de los universitarios. 
Extensión es una forma de relación entre la universidad y la sociedad que puede ser un instrumento de cambio al acercar la universidad a los sectores más desfavorecidos, promover su desarrollo y, en este sentido, establece una relación dialéctica entre la investigación, la docencia y la acción concreta. De este modo, las actividades de extensión deben tener como objetivo prioritario "el apoyo solidario para la resolución de los problemas de exclusión y discriminación social, de tal modo que se dé la voz a los grupos excluidos y discriminados" (Da Souza Santos, 2005:92).

Para lograr un auténtico relevamiento de las necesidades y potencialidades de una comunidad resulta necesario establecer nexos con las asociaciones de la sociedad civil. El contacto directo con estas asociaciones facilita una mirada estratégica sobre las capacidades económicas y productivas de la localidad y proporciona la base necesaria para la articulación de las acciones directas. El presente trabajo apunta también a resolver una de las dificultades que suelen encontrar los programas de extensión universitaria: el hecho de no estar articulados en una propuesta institucional global, lo que podría resultar en la discontinuidad de los proyectos, falta de conexión entre grupos que realizan actividades semejantes, disociación respecto de la docencia y la investigación, carencia de financiamiento adecuado y evaluación regular.

Es por ello que se hace imprescindible incorporar curricularmente a las prácticas sociales universitarias con carácter obligatorio, a través de un profundo debate que vaya más allá de lo administrativo y que implique un verdadero cambio en la cultura institucional de nuestras universidades con el fin de hacer realidad su función social y aportar a un proceso educativo más integral. Así gestado dicho programa, podrá atender a las siguientes instancias: articulación con las organizaciones de la sociedad civil, diagnóstico de potencialidades y necesidades particulares de la localidad en que se inserta, producción de conocimiento, investigación social, articulación de proyectos, abordaje interdisciplinario y acción social directa.

\section{Referencias bibliográficas}

Basabe, L.; Cols, E. y Feeney, S. (2004). Los componentes del contenido escolar. Ficha de la cátedra de Didáctica I. Oficina de Publicaciones de la Facultad de Filosofía y Letras, UBA.

Camillioni, A. (2009). III Congreso de Extensión Universitaria. Santa Fe, 20, 21 y 22 de mayo. Panel: Incorporación curricular de la extensión.

Campus Compact (1999). Higher education in service to the nation. Providence, RI: Campus Compact.

Davini, M.C. (2008). Métodos de Enseñanza: didáctica general para maestros y profesores $\left(1^{\circ} \mathrm{ed}\right.$.). Buenos Aires: Santillana.

Díaz Barriga, F. (2003). Cognición situada y estrategias para el aprendizaje significativo. Revista Electrónica de Investigación Educativa. Disponible en: http://redie. uabc.mx/redie/article/view/85 (consultado el 25 de junio de 2009).

Eyler, J. \& Giles, D. (1999). Where's the learning in service-learning? San Francisco: Jossey-Bass Publishers.

Folgueiras Bertomeu, P. (2013). Aprendizaje y Servicio: Estudio del Grado de satisfacción de estudiantes universitarios. Revista de Educación 362. Facultad de Pedagogía. Universidad de Barcelona.

Folgueiras Bertomeu, P.; González, E. y Juliá, B. (2010). Ciudadanía, participación y aprendizaje y servicio: del centro educativo a la comunidad. Revista Científica Tzhoecoen, III (5), 92-105. Edición especial. Universidad Sr. De SIPAN. Chipalco, Perú. Honnet-Porter, E. \& Poulsen, S. (1989). Principles of good practice for combining service and learning. Wingspread Special Report. Racine, WI: The Johnson Foundation.

Kolb, D. A. (1984). Experiential learning: Experience as the source of learning and development. New Jersey: Prentice-Hall.

Frondizi, R. ([1971] 2005). La universidad en un mundo de tensiones. Cap. 4 "Misión Social: El Sentido dinámico de la misión social". Buenos Aires: Eudeba. Sousa Santos, B. de (2005). La Universidad en el Siglo XXI. Buenos Aires: Miño y Dávila.

Tapia, M. N. (2003). Servicio y Solidaridad en Español: Una cuestión terminológica o un problema conceptual. En Perold, H.; Sherraden, M. and Stroud, S. (Eds.). Servicio Cívico y Voluntariado. EI Servicio Cívico y el Voluntariado en el Siglo XXI (Service Enquiry en Español). Johannesburg: Global Service Institute, USA-Volunteer and Service Enquiry Southern Africa. 